\title{
Electron Vortex Beams and Their Control
}

\author{
Ken Harada ${ }^{1,2, *}$, Teruo Kohashi ${ }^{2}$ and Masanari Koguchi ${ }^{2}$ \\ ${ }^{1}$ CEMS, RIKEN (The Institute of Physical and Chemical Research), Hatoyama, Saitama 350-0395, Japan \\ ${ }^{2}$ Research and Development Group, Hitachi, Ltd., Hatoyama, Saitama 350-0395, Japan
}

\begin{abstract}
Electron vortex beams are considered as probes for next generation electron beam instruments with unprecedented measurement capabilities because vortex beams carry intrinsic orbital angular momenta. In order to clarify the performance of vortex beams, we generated vortex beams by using fork-shaped gratings manufactured by focused ion beam instruments. In vortex beam generation experiment, we found that shapes and sizes of grating openings were superimposed on rings of diffraction spots, typical behavior of vortex beams. This paper discusses experimental results on the generation and control of vortex beams by changing grating opening shapes and sizes. [doi:10.2320/matertrans.MI201901]
\end{abstract}

(Received March 26, 2019; Accepted April 26, 2019; Published June 14, 2019)

Keywords: vortex beam, helical wavefront, fork-shaped grating, orbital angular momentum, topological number

\section{Introduction}

In coherent optics, phase distributions of propagating waves are uniquely determined. The equi-phase plane is called a wavefront and its shape is used to classify types of propagating waves, for example, plane waves, spherical waves, and conical waves. On the other hands, there are waves whose phase distributions are not determined uniquely because they have singular points in the distributions. For example, vortex beams have spiral-shaped waverfronts around the axis of the singular point and their phase distributions have a phase difference of $2 \pi$ or integral multiple of $2 \pi$ when the azimuthal angle is rotated around the singular point. Since existence of singular points is independent of wavefront shapes, vortex beam generation is possible in all the waves of the above classification.

Figure 1 shows waves classified by wavefront shapes and formed vortex beams: Figures 1(a), (b), and (c) are a plane wave, a spherical wave, and a conical wave, respectively, and Figs. 1(d), (e), and (f) are respective helical waves (vortex beams). We note that distortions of the wavefront are concentrated at the core of vortices, i.e., singular points. The orbital angular momentum is inherent in the core and distortions are expressed in terms of topological numbers $n$. This topological number, also called as topological charge, corresponds to the helical number of the spiral shape of the wavefront, and the positive/negative of the topological number corresponds to the right-/left-handed winding helicity of the wavefront.

In optics, optical wave vortex beams are called Laguerre Gaussian beams, ${ }^{1-4)}$ "optical vortex ("hikari-uzu" in Japanese)". ${ }^{5)}$ Since optical waves propagate while keeping orbital angular momenta along the propagation direction, forces can be exerted in the direction perpendicular to the equi-phase plane (wavefront), resulting in releasing momenta to the targets irradiated by the beam. The following manipulation techniques have been practically realized: optical tweezers for controlling particles of a cell size, ${ }^{6-9)}$ laser processing techniques for industrial applications, ${ }^{10-12)}$ super-resolution micro-spectroscopy, ${ }^{13)}$ and quantum information using orbital

*Corresponding author, E-mail: kharada@ riken.jp (a)

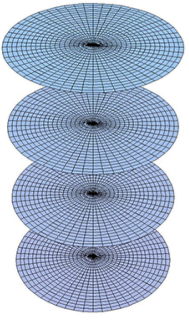

(b)

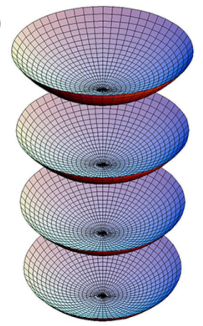

(d)

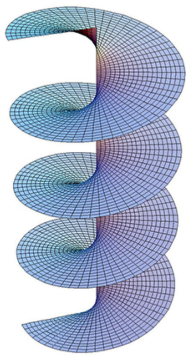

(e)

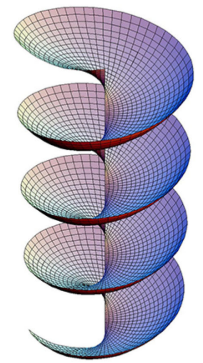

(c)

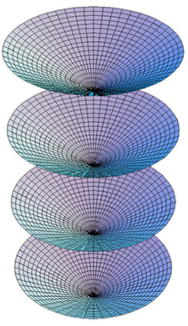

(f)

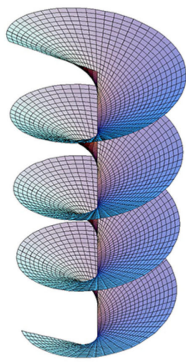

Fig. 1 Classification of the wave by shape of wavefronts: (a) plane wave, (b) spherical wave, (c) conical wave, (d) plane helical wave (vortex beam), (e) spherical helical wave, and (f) conical helical wave.

angular momentum as qubit. ${ }^{14,15)}$ Furthermore, in the X-rays field, new technical developments are expected in studying physical properties of the materials, for example, observation of magnetic states and three-dimensional structure in an atomic scale. $^{16-19)}$

Similarly, in electron optics, electron vortex beams ${ }^{20-23)}$ can carry angular momenta while propagating; therefore, new application fields are expected by using electron beams probes. In observation of magnetic structure in specimens, conventional electron beams have disadvantages of not being able to detect magnetic flux perpendicular to the specimen plane (parallel to the electron beam); in contrast, electron vortex beams have a possibility of detecting magnetic flux perpendicular to the specimen plane owing to its angular momentum in addition to in-plane components (perpendicular to the electron beam) of magnetic flux owing to the Lorenz force. ${ }^{24-26)}$ Furthermore, electron vortex beams have possibilities of being applied to microfabrication and magnetization control. These possible applications indicate 
that electron vortex beams and extended other electron beams such as Bessel beam can become next generation electron beam technologies. ${ }^{27-30)}$

\section{Theoretical}

\subsection{Generation of vortex beams}

Generating electron vortex beams have been realized in the following three methods: fork-shaped grating method, phaseplate method, and magnetic rod method.

In the fork-shaped grating method, gratings with an edge dislocation lattice are irradiated by incident plane electron waves. The diffraction waves passing through the forkshaped grating automatically generate vortex beams. One of the diffraction waves is selected as a vortex beam. ${ }^{21,22)}$ In the phase plate method, thin phase plates with spiral-shaped thickness distribution are installed in the optical system and irradiated with plane electron waves; then transmitted waves have helical wavefronts as vortex beams. ${ }^{23)}$ In the magnetic rod method, the magnetic lines of force from one end of the rod interacts with electron waves through the AharonovBohm effect; ${ }^{31)}$ then electron waves passed through the rod are changed to vortex beams. ${ }^{32,33)}$

Among these methods, we adopted the fork-shaped grating method in our experiment because of simplicity and accuracy. The phase-plate method is difficult to apply to electron microscope observation because electron wavelength is shorter than the atomic size leading to a difficulty in manufacturing thin-film phase-plates with accurate spiral shapes. The magnetic rod method has a difficulty for a practical use because of shadowing of electron beams caused by the magnetic rod, which makes it difficult to image coherent interference states. In the following we discuss vortex beams generated by using fork-shaped gratings.

Figure 2 shows a schematic diagram of vortex beam generation. When a coherent plane wave is irradiated on a fork-shaped grating, waves after passing through the grating are diffracted in left and right directions, and become vortex beams having opposite helicities. This method requires an optical system in which coherent electron beams can irradiate on an area wider than the grating size. An optical system for small angle electron diffraction (SmAED) $)^{34-36)}$ satisfies this requirement and is used in the present experiments. The order numbers of diffraction waves in Fig. 2 correspond to the topological number of the vortex beam. The left and right diffraction waves with different positive and negative diffraction angles have the positive and negative topological numbers.

\section{Incident electron beam (Plane wave)}

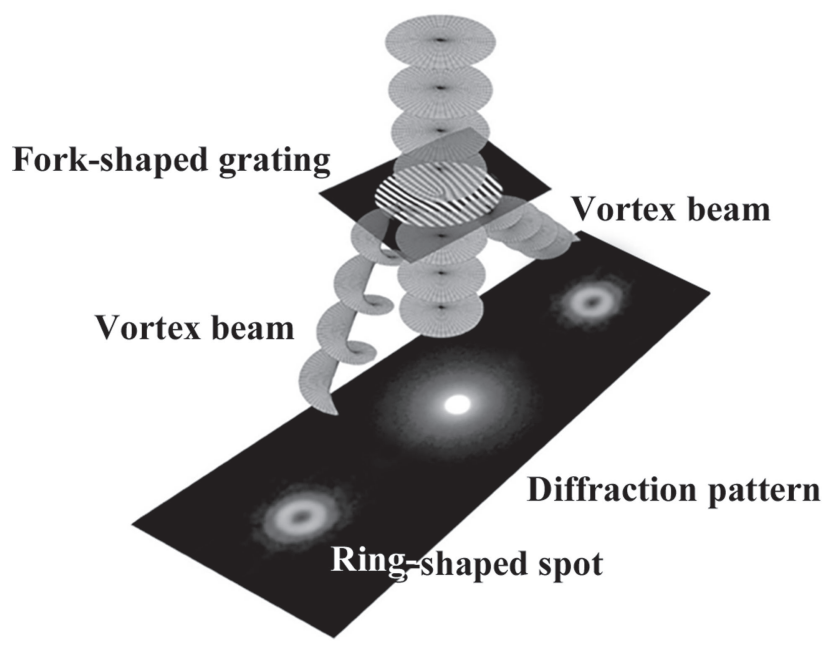

Fig. 2 Schematic diagram of vortex beam generation using fork-shaped grating.

\subsection{Fork-shaped grating}

Vortex beam of plane-wave type can be generated by using fork-shaped gratings. The gratings were fabricated on the basis of the interference fringes made of a plane wave in the $\mathrm{x}-\mathrm{y}$ plane $\varphi_{\mathrm{p}}(x, y)$ and a plane-helical wave $\varphi_{\mathrm{ph}}(x, y)$. These waves are given by

$$
\begin{aligned}
\varphi_{p}(x, y) & =\exp \left[-i \frac{2 \pi}{\lambda} \alpha x\right], \\
\varphi_{p h}(x, y) & =\exp [\operatorname{in} \theta]=\exp \left[\operatorname{in} \tan ^{-1}\left[\frac{y}{x}\right]\right],
\end{aligned}
$$

where $\lambda$ is the wavelength, $\alpha$ is the relative angle between the two waves $\varphi_{\mathrm{p}}(x, y)$ and $\varphi_{\mathrm{ph}}(x, y), n$ is the topological number, and $\theta$ is the azimuthal angle.

Then the intensity distribution $I(x, y)$ of the interference fringes can be expressed by

$$
I(x, y)=2+2 \cos \left[\frac{2 \pi}{\lambda} \alpha x+n \tan ^{-1}\left[\frac{y}{x}\right]\right] .
$$

The interference fringes expressed by eq. (3) include the edge dislocation with the $n$th order at the origin of the grating, $(x, y)=(0,0)$. The grating was fabricated based on eq. (3).

Figure 3 shows calculated intensity distributions of the fork-shaped gratings given by eq. (3). The edge dislocations with the order number $n$ shown at the center of the gratings correspond to the topological number $n$, when the vortex beams are generated using these gratings.

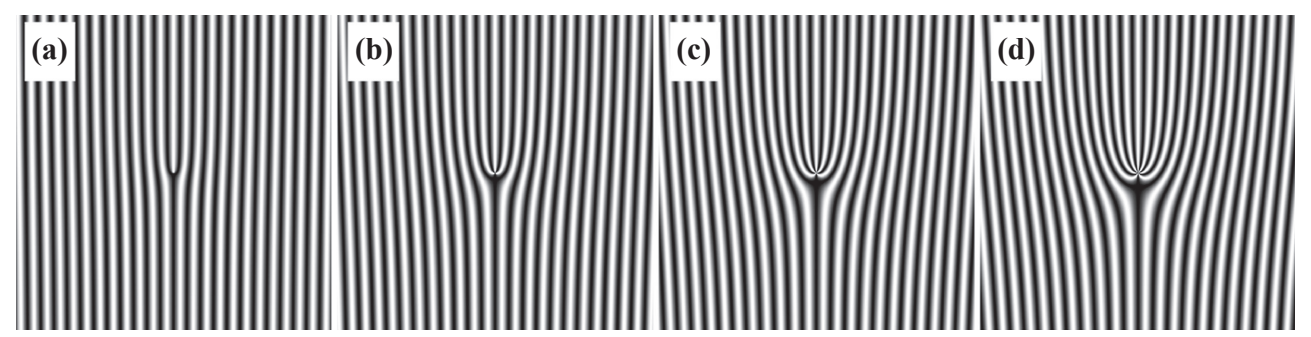

Fig. 3 Calculated fork-shaped gratings with the topological number $n$ : (a) $n=1$, (b) $n=3$, (c) $n=5$, and (d) $n=7$. 

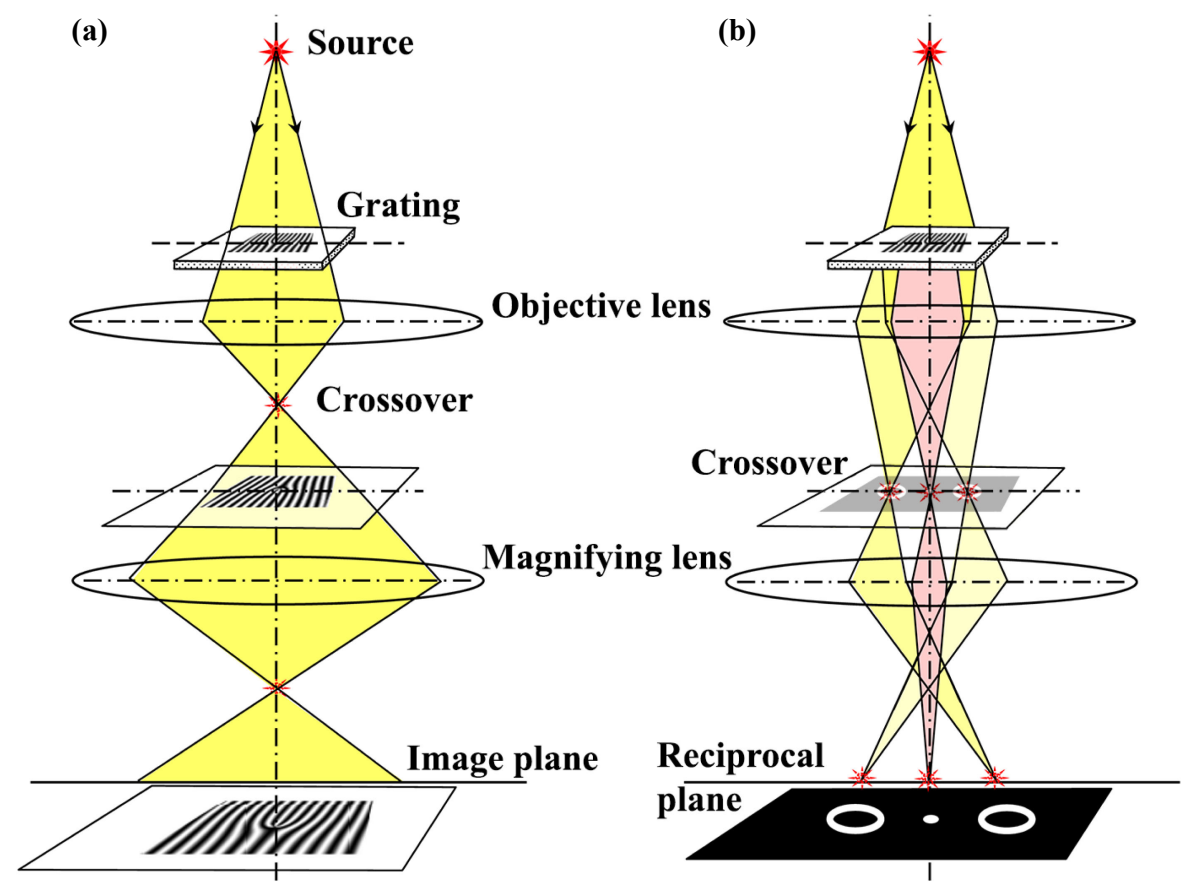

Fig. 4 An optical system for vortex beam observation: (a) imaging mode for fork-shaped gratings and (b) small angle electron diffraction (SmAED) mode for observing the vortex beams.

\section{Experimental}

\subsection{Optical systems}

Generation of vortex beams is confirmed through annular ring-shaped diffraction spots on the diffraction pattern. Figure 4(a) shows an optical system for directly observing the fork-shaped gratings and Fig. 4(b) shows an SmAED optical system for confirmation of vortex beams. Fork-shaped gratings are installed at the specimen position of the electron microscope. Since an averaged grid spacing of $400 \mathrm{~nm}$ is much larger than the ordinary crystalline lattice spacing by three orders of magnitude or more, an SmAED optical system with a camera length of $150 \mathrm{~m}$ or more is required. ${ }^{37)}$

\subsection{Experimental setup}

The fork-shaped gratings having an averaged grid spacing of $400 \mathrm{~nm}$ were made of a silicon nitride $\left(\mathrm{Si}_{3} \mathrm{~N}_{4}\right)$ membrane of $200 \mathrm{~nm}$ thick by using focused ion beam (FIB) instrument (FB-2100, Hitachi High-Technologies Corp.). A prototype $300-\mathrm{kV}$ field emission electron microscope (H-9000) was used for observation. ${ }^{38)}$ Grating images and diffraction patterns were recorded by a $2 \mathrm{k} \times 2 \mathrm{k}$ pixels charge-coupleddevice camera system (Orius, Gatan, Inc.).

\section{Results and Discussions}

\subsection{Measurement of topological number}

Left panels in Fig. 5 shows the fork-shaped gratings having the $1 \mathrm{st}, 3 \mathrm{rd}, 5 \mathrm{th}$, and 7 th ordered edge dislocations at the center and the right panel shows vortex beams characterized by annular ring-shaped diffraction spots. The diameter of the ring-shaped spots increases with the increase in the order of diffraction and the order of dislocation inside the grating.

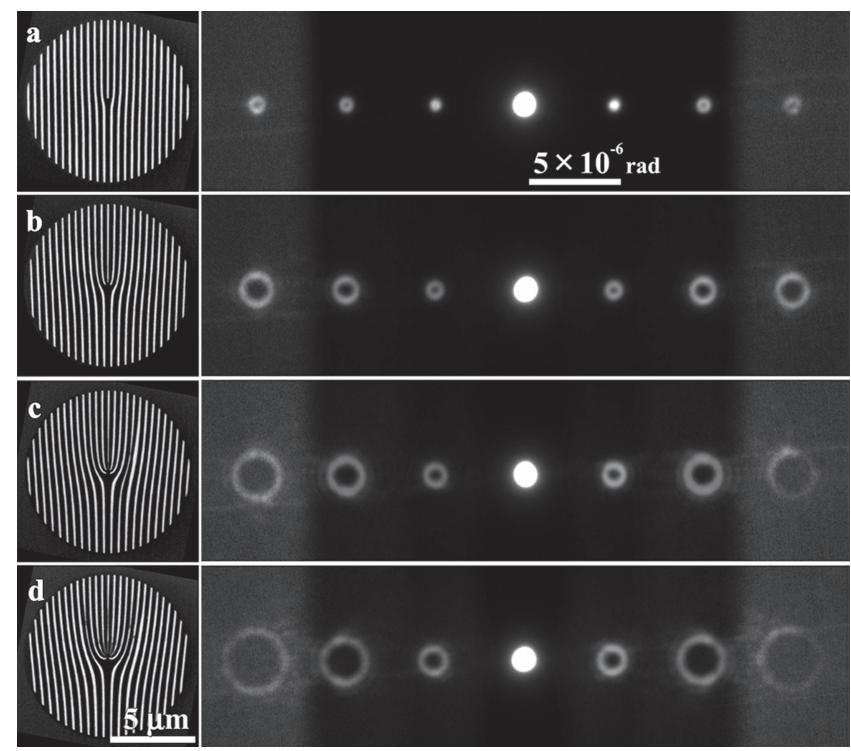

Fig. 5 Fork-shaped gratings and their electron diffraction patterns Topological numbers are as follows: (a) $n=1$, (b) $n=3$, (c) $n=5$, and (d) $n=7$. Ring diameters of diffraction depend on the order of the lattice dislocation and the order of diffraction. Contrast of the diffraction spots in higher order are enhanced for better visualization.

We note that the order of the edge dislocation in the forkshaped grating is equal to the topological number of the generated vortex beam for the lowest order diffraction wave. For higher order diffraction waves, the number of topological charge of the beam is the product of the order number of the dislocation and the degree of the diffraction spot. Then the maximum topological number $n_{\max }$ is \pm 21 , for 3 rd order diffraction spot in the 7 th order dislocation of the fork-grating (see. Fig. 5(d)). 


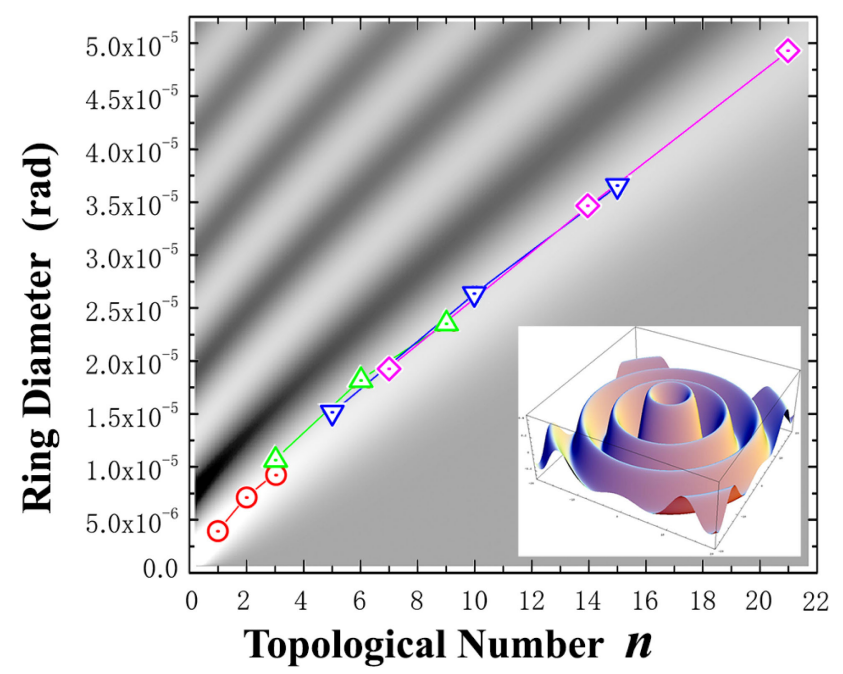

Fig. 6 Relation between the ring diameter and the topological number in terms of the Bessel function of the first kind. Inset is a three-dimensional drawing of the Bessel function of the first kind.

A correlation exists between the topological number and the annular ring diameter of the diffraction spots. When the wave function of the vortex beam $\Psi(r, \theta, z)$ is divided into three eigen functions $R(r), \Phi(\theta)$, and $Z(z)$ through the cylindrical coordinate system $(r, \theta, z)$, eigen functions are given by

$$
\begin{aligned}
\Psi(r, \theta, z) & =R(r) \Phi(\theta) Z(z) \\
& =J_{n}(r) \Phi_{0} \exp ( \pm 2 \pi i n \theta) Z_{0} \exp ( \pm 2 \pi i k z),
\end{aligned}
$$

where $\Phi_{0}$ and $Z_{0}$ are the initial values of each functions. The shape of equi-phase plane -wavefront- of the wave function is characterized by the radial function $R(r)$ in terms of the Bessel function of the first kind, $J_{n}(x) .{ }^{39,40)}$

Figure 6 shows the relation between the ring diameter and the topological number $n$ of the vortex beam. In the background of Fig. 6, values of the Bessel function of the first kind are drawn with the topological number $n$ as a parameter. The ring diameters of the diffraction spots and the lowest order stripe can be fitted to the curve of the Bessel function of the first kind.

\subsection{Control of vortex beams by opening shapes}

Experiments clearly showed that the ring diameter of the diffraction spots are fitted to the curve of the lowest order of the Bessel function of the first kind; however, exact determination of the relation of the diameter to the topological number was difficult, probably because the diameters depend on the opening sizes of the fork-shaped gratings. ${ }^{41,42)}$ Figure 7 shows electron micrographs of circular fork-shaped gratings on the left panels and their electron diffractions on the right panels. For smaller opening sizes, larger diameter diffraction rings were obtained. The electron beam intensities decreased inversely proportional to the square of the opening diameter, and the diameter of annular diffraction spots increased when the opening diameter decreased; as a result, the intensity per unit area of the vortex beam decreased.

Since the opening sizes affect the ring diameters in the diffraction pattern, the opening shape should affects the ring

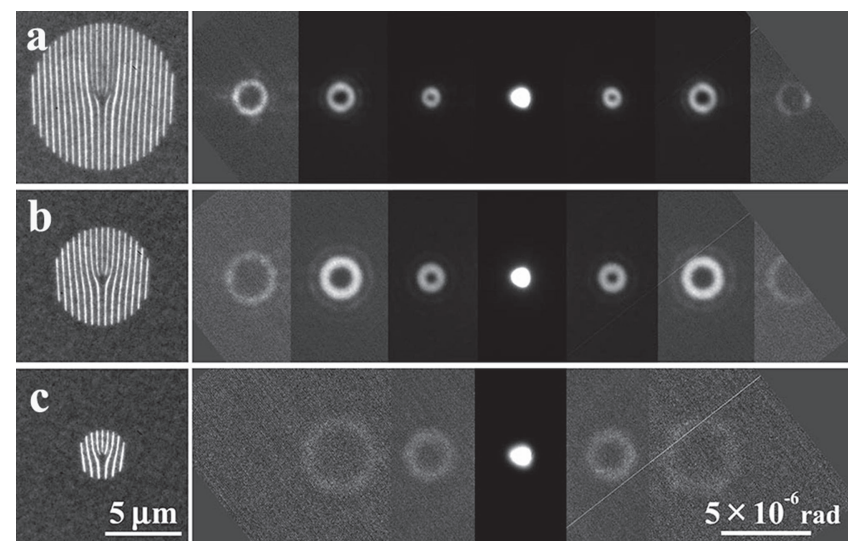

Fig. 7 Ring diameters of diffraction are a function of opening sizes: (a) $10 \mu \mathrm{m}$ in diameter of the opening, (b) $6.7 \mu \mathrm{m}$ in diameter, and (c) $3.3 \mu \mathrm{m}$ in diameter. Contrast of the diffraction spots in higher order are enhanced for better visualization.
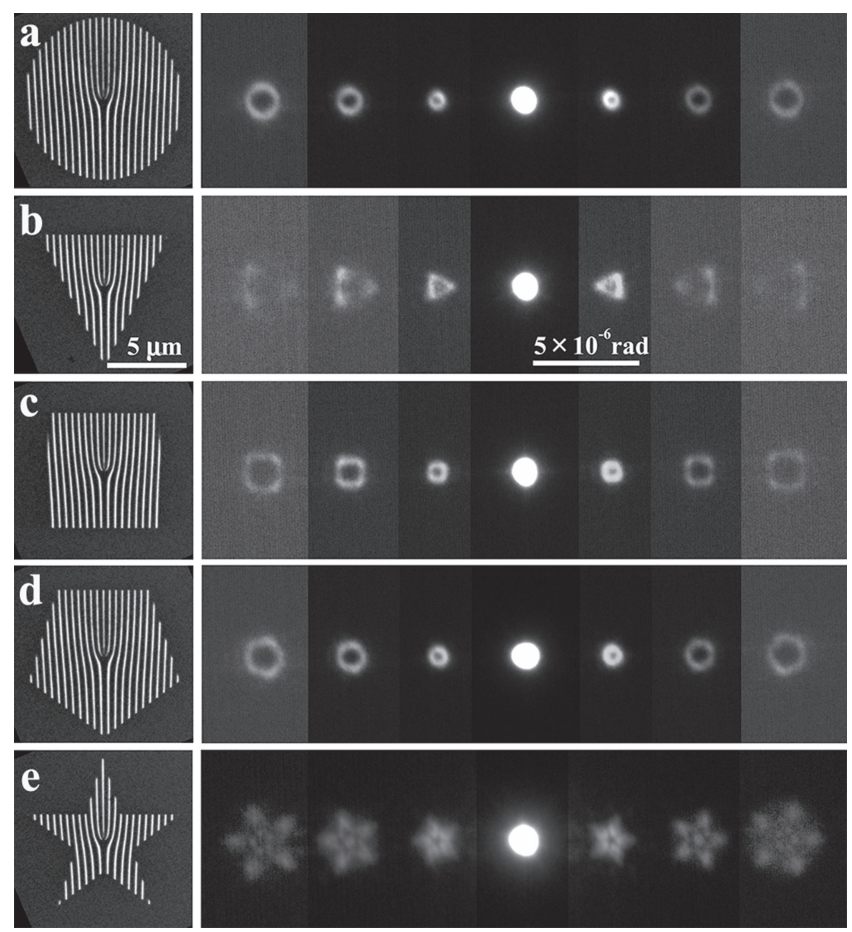

Fig. 8 Effect of opening shapes on diffraction ring shapes: (a) circular opening, (b) triangular opening, (c) square opening, (d) pentagonal opening, and (e) star-shaped opening. The fork-shaped gratings have the third order edge dislocation and their average grid spacing is $400 \mathrm{~nm}$.

shape. To check this hypothesis we observed diffraction patterns by changing opening shapes. Figure 8 shows the results for different shapes: (a) circular shape, (b) triangular shape, (c) square shape, (d) pentagonal shape, and (e) star shape. The left panels show the electron micrographs of the fork-shaped gratings with the third ordered edge dislocation at the center of the openings. The right panels show SmAED patterns whose ring-shaped spots reflected the shapes of the openings. ${ }^{41,42)}$

Diffraction patterns on the right panels show that shapes of ring-shaped diffraction spots reproduced opening shapes. Generally, diffraction patterns from gratings shows simple spots corresponding to the spatial frequency and azimuthal 


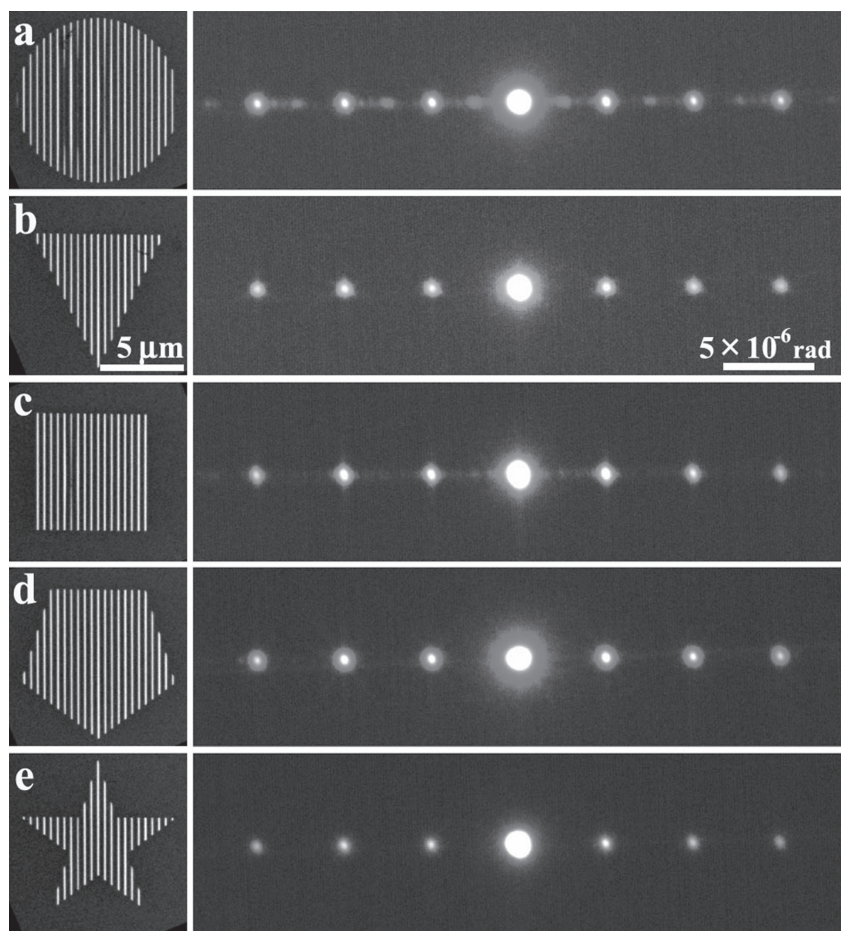

Fig. 9 No effects of opening shape on diffraction spot shapes for simple grating without edge dislocations: (a) circular opening, (b) triangular opening, (c) square opening, (d) pentagonal opening, and (e) star-shaped opening. All the openings generated simple diffraction spots corresponding to the spatial frequency of the simple grating.

directions in the real space in which the grids are arranged. In the present experiments, opening shapes in the real space are reproduced in diffraction ring shapes in the reciprocal space. We note that diffraction patterns show $90^{\circ}$ azimuthal rotation from the opening shape for triangular, pentagonal, and star-shaped openings, hypothesizing existence of relations to Fourier transforms of opening shapes or Fraunhofer diffractions.

To check this hypothesis, we studied the effects of Fraunhofer diffraction from the opening using simple gratings without an edge dislocation, which then do not generate vortex beams. Figure 9 shows the results: all openings created simple diffraction spots corresponding to the spatial frequency of the simple gratings. Comparison of results in Figs. 8 and 9 indicates effects of the interaction between the vortex beams, and the Fraunhofer diffraction waves from the opening shapes in the real space are reflected in the shapes of diffraction spots in the reciprocal space.

Next we simulate the interaction between the helical waves generated by the edge dislocation in the fork-shaped grating and Fraunhofer diffraction waves from the opening shapes. Figure 10 shows the results of two-dimensional Fourier transform for the same opening shapes discussed in Figs. 8 and 9, clearly reproducing the diffraction patterns shown in Fig. 8. This is because the electron waves used in our experiments were sufficiently coherent throughout the $10 \mu \mathrm{m}$ size opening of the grating. These results shown in Figs. 8, 9 and 10 are new findings, and now we have new means of controlling diffraction spots in the reciprocal space from the real space.
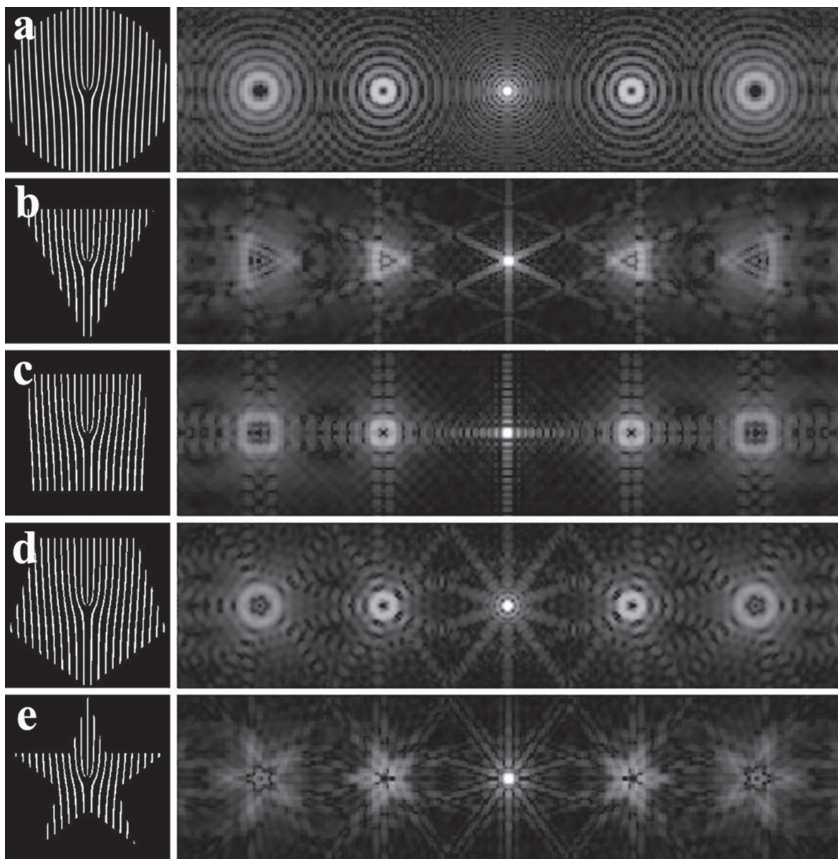

Fig. 10 Two-dimensional Fourier transform results for five opening shapes of the fork-shaped gratings: (a) circular opening, (b) triangular opening, (c) square opening, (d) pentagonal opening, and (e) star-shaped opening. Diffraction patterns in Fig. 8 are clearly reproduced.

\subsection{Profile of the vortex beams}

These findings of strong effects of opening shapes on vortex beams shown in diffraction patterns led us to perform experiments on twisting beam profile observation. Figure 11 shows diffraction patterns from a fork-shaped grating with a diamond-shaped opening for five defocusing distances. The out-of-focus condition was produced by changing the focal length of the objective lens (see, Fig. 4). When the focus condition was changed from under-focus to over-focus -through-focus condition-, the diamond-shaped diffraction spots on the right side rotated in the counterclockwise azimuthal direction and the spots on the left side rotated in the clockwise azimuthal direction. Since the shape of the diffraction spots is diamond-shaped, the azimuthal rotation was clearly visible. ${ }^{41,42)}$

Figure 12 plots the rotation angles of the first, second, and third diffraction spots versus the defocusing distance $\Delta f$. The rotation angle increases (decreased) with the increase in the defocus distance for the right (left) diffraction spots, and lower ordered spots rotated more.

The twisted profile of electron vortex beams was reconstructed by three-dimensional image processing method and the results are shown in Fig. 13:43,44) forty-five observed patterns are stacked in the vertical direction and an electron beam profile (intensity) in the vicinity of the convergence point of the diffraction spot is drawn. Since the left and right diffraction spots are twisted in the opposite azimuthal directions, this twist is not due to Larmor rotation caused by the magnetic field in the electromagnetic lens. The rotation itself can be explained by the Gouy phase. ${ }^{41,42,45-48)}$

On the basis of these experimental results, we confirmed that the infocus condition of the diffraction patterns can be determined precisely through experiments by using the 

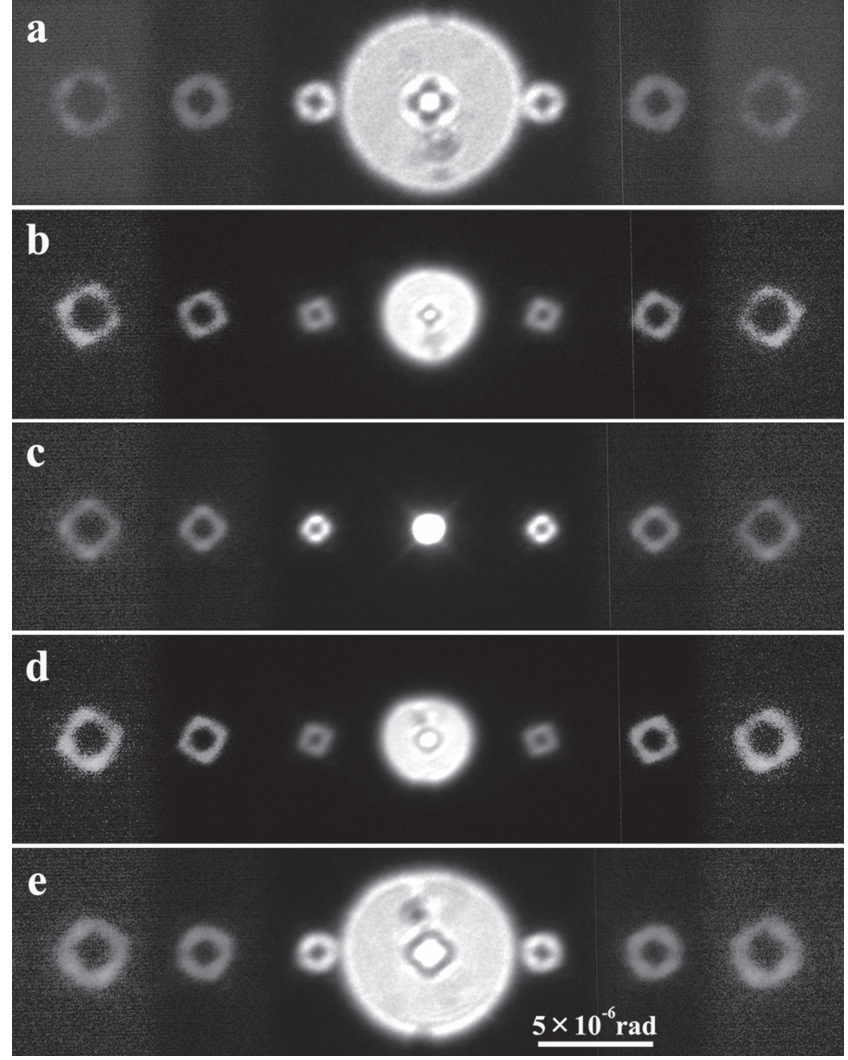

Fig. 11 Changes in diffraction patterns from a fork-shaped grating with a diamond-shaped opening under different defocusing distances $\Delta f$ : (a) $\Delta f=2 \mathrm{~mm}$ (under-focus), (b) $\Delta f=1 \mathrm{~mm}$ (under-focus), (c) $\Delta f=$ $0 \mathrm{~mm}$ (infocus), (d) $\Delta f=-1 \mathrm{~mm}$ (over-focus), and (e) $\Delta f=-2 \mathrm{~mm}$ (over-focus). The diamond-shaped diffraction spots rotate with the defocusing distance changes.

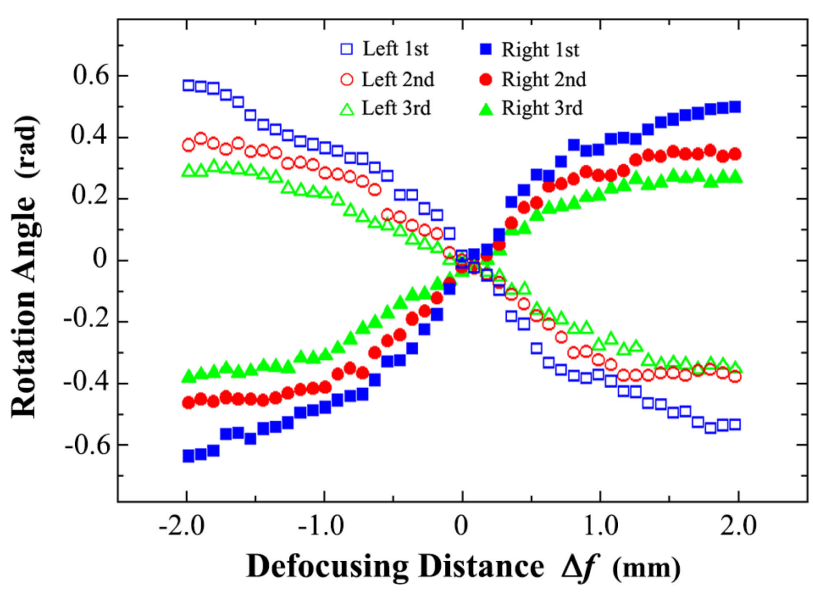

Fig. 12 Azimuthal rotation angles of the first, second and third diffraction spots on both sides versus defocusing distance $\Delta f$.

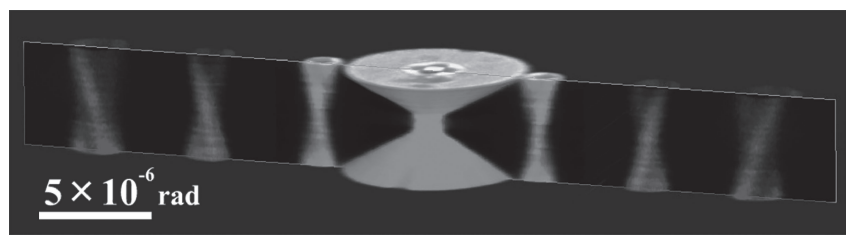

Fig. 13 Three-dimensional reconstruction of diffraction beams. opening shapes reflected on the diffraction spots. We expect that this developed technique will be utilized to determine the infocus condition in the scanning transmission electron microscopy.

\section{Conclusion}

We confirmed generation of electron vortex beams having helical wavefronts from electron beams passing through forkshaped gratings with edge dislocations by observing annular ring-shaped diffraction spots in the small angle electron diffraction (SmAED) optical system. We also succeeded in controlling vortex beams by changing opening shapes and sizes, and grid spacing. We expect that vortex beams carrying orbital angular momenta can be used as next generation electron probes to study unexplored electron properties. We hope to develop new measuring and processing technologies using vortex beams in near future.

\section{Acknowledgement}

The authors would like to thank Mr. T. Iwane, Mr. N. Moriya, and Ms. M. Yamane of Hitachi, Ltd. for their technical support.

\section{REFERENCES}

1) H. Kogelnik and T. Li: Proc. IEEE 54 (1966) 1312-1329.

2) L. Allen, M.W. Beijersbergen, R.J.C. Spreeuw and J.P. Woerdman: Phys. Rev. A 45 (1992) 8185-8189.

3) L. Allen, S.M. Barnett and M.J. Padgett (eds.): Optical Angular Momentum, (CRC Press Taylor \& Francis Group, London, 2003) pp. 21-26.

4) S. Franke-Arnold, L. Allen and M.J. Padgett: Laser Photon. Rev. 2 (2008) 299-313.

5) Y. Miyamoto and A. Wada: Optics 35 (2006) 618-624 (in Japanese).

6) H. He, M.E.J. Fries, N.R. Heckenberg and H. Rubinsztein-Dunlop: Phys. Rev. Lett. 75 (1995) 826-829.

7) Q. Zhan and J.R. Leger: Opt. Express 10 (2002) 324-331.

8) N.B. Simpson, K. Dholakia, L. Allen and M.J. Padgett: Opt. Lett. 22 (1997) 52-54.

9) M.E.J. Friese, T.A. Nieminen, N.R. Heckenberg and H. RubinszteinDunlop: Nature 394 (1998) 348-350.

10) A.V. Nesterov, V.G. Niziev and V.P. Yakunin: J. Phys. D 32 (1999) 2871-2875.

11) V.G. Niziev and A.V. Nesterov: J. Phys. D 32 (1999) 1455-1461.

12) K. Toyoda, K. Miyamoto, N. Aoki, R. Morita and T. Omatsu: Nano Lett. 12 (2012) 3645-3649.

13) B. Harke, J. Keller, C.K. Ullal, V. Westphal, A. Schönle and S.W. Hell: Opt. Express 16 (2008) 4154-4162.

14) A. Mair, A. Vaziri, G. Weihs and A. Zeilinger: Nature 412 (2001) 313316.

15) J. Leach, M.J. Padgett, S.M. Barnett, S. Franke-Arnold and J. Courtial: Phys. Rev. Lett. 88 (2002) 257901.

16) Y. Takahashi, A. Suzuki, S. Furutaku, K. Yamauchi, Y. Kohmura and T. Ishikawa: Phys. Rev. B 87 (2013) 121201.

17) Y. Kohmura, V. Zhakhovsky, D. Takei, Y. Suzuki, A. Takeuchi, I. Inoue, Y. Inubushi, N. Inogamov, T. Ishikawa and M. Yabashi: Appl. Phys. Lett. 112 (2018) 123103.

18) S. Matsuba, K. Kawase, A. Miyamoto, S. Sasaki, M. Fujimoto, T. Konomi, N. Yamamoto, M. Hosaka and M. Katoh: Appl. Phys. Lett. 113 (2018) 021106.

19) Y. Taira and M. Katoh: Phys. Rev. A 98 (2018) 052130.

20) M. Uchida and A. Tonomura: Nature 464 (2010) 737-739.

21) J. Verbeeck, H. Tian and P. Schattscheider: Nature 467 (2010) 301-304.

22) B.J. McMorran, A. Agrawal, I.M. Anderson, A.A. Herzing, H.J. Lezec, 
J.J. McCelland and J. Unguris: Science 331 (2011) 192-195.

23) K. Saitoh, Y. Hasegawa, N. Tanka and M. Uchida: J. Electron Microsc 61 (2012) 171-177.

24) K.Y. Bliokh, M.R. Dennis and F. Nori: Phys. Rev. Lett. 107 (2011) 174802.

25) P. Schattschneider, M. Stöger-Pollach and J. Verbeeck: Phys. Rev. Lett. 109 (2012) 084801.

26) P. Schattschneider, S. Loffler, M. Stöger-Pollach and J. Verbeeck: Ultramicroscopy 136 (2014) 81-85.

27) B.J. McMorran, A. Agrawal, P.A. Ercius, V. Grillo, A.A. Herzing, T.R Harvey, M. Link and J.S. Pierce: Phil. Trans. R. Soc. A 375 (2017) 20150434.

28) K. Saitoh, Y. Hasegawa, K. Hirakawa, N. Tanaka and M. Uchida: Phys. Rev. Lett. 111 (2013) 074801.

29) V. Grillo, E. Karimi, G.C. Gazzadi, S. Frabboni, M.R. Dennis and R.W. Boyd: Phys. Rev. X 4 (2014) 011013.

30) V. Grillo, G.C. Gazzadi, E. Karimi, E. Mafakheri, R.W. Boyd and S. Frabboni: Appl. Phys. Lett. 104 (2014) 043109.

31) A. Tonomura: Rev. Mod. Phys. 59 (1987) 639-669.

32) A. Béché, R. Van Boxem, G. Van Tendeloo and J. Verbeeck: 18th International Microscopy Congress (IMC18), (Prague, Czech, September 7-12, 2014) IT-1-P-1486 (two pages).

33) A.M. Blackburn and J.C. London: Ultramicroscopy 136 (2014) 127143.

34) T. Yoshida, J. Endo, H. Kasai, K. Harada, T. Matsuda and A. Tonomura: J. Appl. Phys. 85 (1999) 1228-1230.

35) T. Koyama, K. Takayanagi, Y. Togawa, S. Mori and K. Harada: AIP Adv. 2 (2012) 012195
36) H. Nakajima, A. Kotani, K. Harada, Y. Ishii and S. Mori: Microscopy 65 (2016) 473-478.

37) K. Harada: Appl. Phys. Lett. 100 (2012) 061901.

38) T. Kawasaki, T. Matsuda, J. Endo and A. Tonomura: Jpn. J. Appl. Phys. 29 (1990) L508-L510.

39) P. Schattschneider and J. Verbeeck: Ultramicroscopy 111 (2011) 14611468.

40) K. Saitoh, Y. Hasegawa and M. Uchida: Kenbikyo 48 (2013) 39-46 (in Japanese).

41) K. Harada, T. Kohashi and T. Iwane: Microsc. Microanal. 20 (2014) 273-275.

42) K. Harada, T. Kohashi and T. Iwane: 18th International Microscopy Congress (IMC18), (Prague, Czech, September 7-12, 2014) IT-11-P1673 (two pages).

43) K. Harada: Proc. NIMS Conference 2015 -Materials Innovation Driven by Advanced Characterization-, (Tsukuba, Ibaraki, July 14-16, 2015) TS4-3, p. 45.

44) K. Harada, T. Kohashi and T. Iwane: Microsc. Microanal. 21 (2015) $669-670$.

45) C.R. Gouy and C.R. Hebd: Seances Acad. Sci. 110 (1890) 1251.

46) G. Guzzinati, P. Schattscheider, K.Y. Bliokh, F. Nori and J. Verbeeck: Phys. Rev. Lett. 110 (2013) 093601

47) Y. Hasegawa, K. Saitoh, N. Tanka and M. Uchida: J. Phys. Soc. Jpn. 82 (2013) 073402.

48) P. Schattschneider, Th. Schachinger, M. Stöger-Pollach, S. Löffler, A. Steiger-Thirsfeld, K.Y. Bliokh and F. Nori: Nat. Commun. 5 (2014) 4586 . 